\title{
Image-based high strain-rate testing for the characterization of viscoplasticity
}

\author{
Pascal Bouda ${ }^{1,2, *}$, Bertrand Langrand ${ }^{1}$, Delphine Notta-Cuvier ${ }^{2}$, Eric Markiewicz ${ }^{2}$, and Fabrice Pierron ${ }^{3}$ \\ ${ }^{1}$ French Aerospace Research Center (ONERA), Dept. Materials and Structures, Lille - France \\ ${ }^{2}$ University of Valenciennes, LAMIH UMR CNRS 8201, Valenciennes - France \\ ${ }^{3}$ University of Southampton, Faculty of Engineering and the Environment, Southampton - UK
}

\begin{abstract}
The present work aims at identifying an elastic-viscoplastic material behaviour over a wide strain and strain-rate range (up to 0.1 and $1000 \mathrm{~s}^{-1}$ respectively), using the so-called Virtual Fields Method. To define the experimental campaign, a design process has been set. This relies on the numerical optimization of the setup - notably the specimen shape ? with respects to user-defined criteria. Finally, the selected configuration ensures an accurate and robust identification of material parameters.
\end{abstract}

\section{Introduction}

Ultra-High-Speed imaging is nowadays widely used by the experimental community in dynamic testing. As optical devices are continuously being improved (in terms of resolution, interframe rate, e.g.), the combination with a full-field measurement technique is more and more suitable for material behaviour characterization at high strain rate. Among the available inverse methods, the Dynamic Virtual Fields Method (DVFM) [1] enables - based of the Principle of Virtual Work (PVW) - the identification of material parameters with the sole knowledge of the strain and the acceleration fields (Equation 1). In particular, the DVFM does not require the knowledge of any external loads, this avoiding the use of an intrusive sensor (e.g. accelerometer, strain gauge, ....).

$$
-\underbrace{\int_{V} \boldsymbol{\sigma}: \boldsymbol{\varepsilon}^{*} d V}_{W_{i n t}^{*}}=\underbrace{\int_{V} \rho \boldsymbol{\gamma} \cdot \mathbf{u}^{*} d V}_{W_{a c c}^{*}}
$$

Inertial impact tests have been recently proposed to take advantage of the DVFM for material testing at high strain rate [2]. However, the selected configuration was defined without an in-depth analysis of its features. As non-linear viscoplastic behaviour might need a lot of material parameters to be described, it is necessary to explore more extensively the design space in order to optimize the procedure of identification, in particular with a reduced number of involved tests and an effective exploitation of the available data. This design space depends on several parameters, some of them being directly linked to the processing toolchain (full-field measurement technique, VFM toolchain features).
The present work aims at optimizing the aforementioned impact test to characterize the elastic-viscoplastic behaviour of a metallic material over a wide strain and strain-rate range (up to 0.1 and $1000 \mathrm{~s}^{-1}$ respectively), using the DVFM. Two criteria govern the selection: (1) - the strain and the strain-rate range (2) - the identifiability of the parameters.

\section{Assessment of a test configuration}

In this work, Finite Element Analysis (FEA) is used as a basis to assess the two selected design criteria. Contrary to an approach with real tests, the numerical models enable the analysis of the test parameters influence in an iterative procedure.

The first criterion is assessed based on FE results. A bidimensionnal histogram (Figure 1) collects the mechanical states which undergo viscoplastic deformation for each measurement point (i.e. the mesh elements). More precisely, the viscoplastic spectrum (cumulated plastic strain, $p$, vs. equivalent plastic strain rate, $\dot{p}$ ) which is covered by a test configuration is plotted.

To take into account experimental biases in the identification toolchain (full-field measurement features, digitization process, camera noise, dynamic range, ... ), virtual images are generated using a new gradient-based algorithm. This has already been addressed for another cases under quasi-static [3, 4] and dynamic loadings [5] but the proposed methods suffered from critical drawbacks which could have jeopardized their use in ultra high-speed imaging. Finally, the simulated images are input in the VFM toolchain to assess the second criterion.

*e-mail: pascal.bouda@onera.fr 


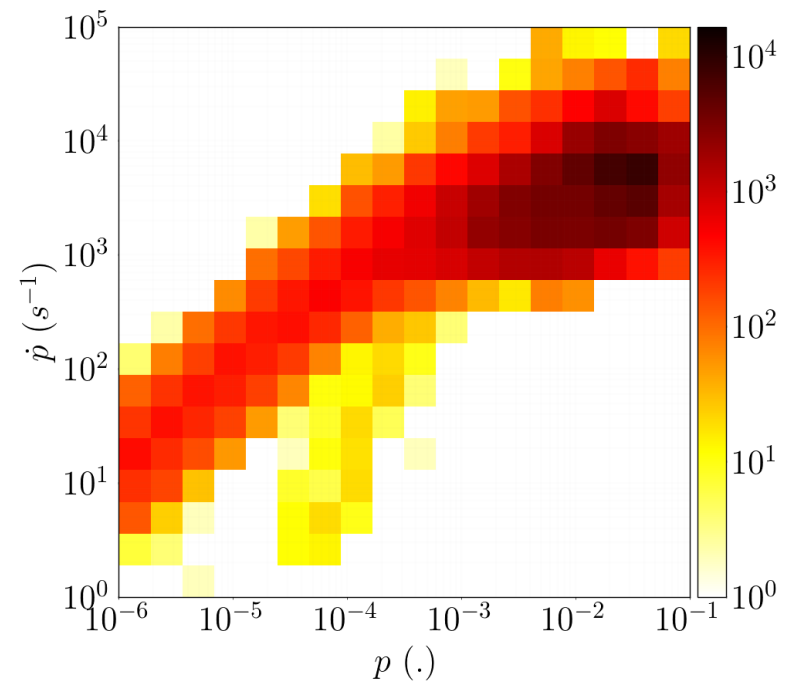

Fig. 1. Example of a viscoplasticity histogram.

\section{Optimization of the test configuration}

This enhancement process notably relies on the topological optimization of the specimen inner geometry $S_{s}^{\text {int }}$. For given impact conditions (projectile mass $M_{p}$ and velocity $V_{p}$ ), it has to promote the development of viscoplasticity over the whole targeted spectrum. To do so, geometric singularities (e.g. holes, notches) with various properties are manufactured at different locations in the specimen to enhance stress concentrations. However, this shrinking process may jeopardize the identification insofar as it decreases the specimen surface area for a given field-ofview. Thus, the best solution is a compromise between the required viscoplastic flow and the robustness of the identification procedure. For instance, the specimen inner geometry $S_{s}^{\text {int }}$ which enables the most robust identification with the DVFM w.r.t camera noise may minimize the following functionnal:

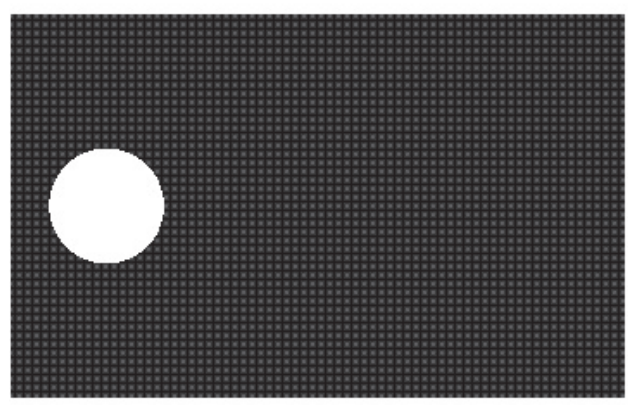

(a)

$$
\Phi\left(S_{s}^{\text {int }}\right)=\sum_{i}\left\|\mathbf{X}^{\mathbf{r e f}}-\mathbf{X}^{\mathrm{mes}}\left(S_{s}^{i n t}\right)[i]\right\|
$$

where $\mathbf{X}^{\text {ref }}$ denotes the reference values of the identified parameters, $i$ is the index of the trial sets of virtual images polluted by a random noise and $\mathbf{X}^{\mathbf{m e s}}\left(S_{s}^{i n t}\right)[i]$ the parameters which are extracted from each set of images by the DFVM (e.g. Figure 2).

To restrict the optimization problem, the specimen inner geometry $S_{s}^{\text {int }}$ is driven by an evolution law for the geometric singularities. The Figure 2 shows the undeformed and the deformed state of a specimen, before extracting the kinematic fields with the grid method. As a starting geometry, a circular hole was considered in the vicinity of the impact zone. Whereas the accumulation of geometric singularities may jeopardize the identification, it also strengthens the stress concentrations, hence resulting a wider viscoplastic spectrum. Thus, a compromise is found w.r.t the topological optimization cost function.

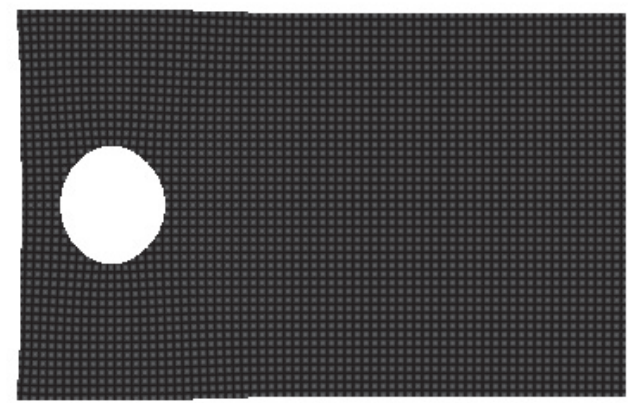

(b)

Fig. 2. Illustration of the image simulator - (a) initial state - (b) final state. 


\section{Conclusion}

In this study, new tests configurations are introduced to characterize viscoplastic behaviours at high-strain rates. This is based on the Dynamic Virtual Fields Method (DFVM) which is input with full-field kinematic maps (strains and accelerations) to extract the material parameters. As no standards have been defined, a fully computational approach is addressed to improve the features of a starting test configuration. Then, the future work is to assess the optimization results with a test campaign in real conditions.

\section{References}

1. R. Moulart, F. Pierron, S. Hallett, M. Wisnom, Experimental Mechanics 51, 509 (2011)

2. F. Pierron, H. Zhu, C. Siviour, Philosophical Transactions of the Royal Society of London A: Mathematical, Physical and Engineering Sciences 372 (2014)

3. M. Rossi, F. Pierron, International Journal of Solids and Structures 49, 420 (2012)

4. M. Rossi, P. Lava, F. Pierron, D. Debruyne, M. Sasso, Strain 51, 206 (2015)

5. B. Lukić, D. Saletti, P. Forquin, Philosophical Transactions of the Royal Society of London A: Mathematical, Physical and Engineering Sciences 375 (2016) 
